\title{
Design and Upgrade of a Compact Imaging System for the APS Linac Bunch Compressor
}

\author{
B. Yang, E. Rotela, S. Kim, R. Lill, and S. Sharma \\ Argonne National Laboratory, 9700 South Cass Avenue, Argonne, IL 60439
}

\begin{abstract}
We present the design, performance, and recent upgrade of a high-resolution, highcharge-sensitivity imaging camera and beam position monitor (BPM) system for the APS linac beam profile measurement. Visible light is generated from the incoming electron beam using standard YAG or optical transition radiation (OTR) converter screens. Two CCD cameras share the light through a beam splitter, each with its own imaging optics. Normally, one camera is configured with high magnification and the other with large field of view. In a different lens configuration, one of the cameras focuses at the far field, allowing the measurement of beam divergence using an OTR screen, while the other camera simultaneous measures the beam size. A four-position actuator was installed recently to provide the option of two screens, a wakefield shield, and an in situ calibration target. A compact S-band beam position monitor electrode was designed to mount directly on the flag. The BPM rf circuit was fabricated from a machinable ceramic (MACOR) cylinder substrate, and the copper electrodes were deposited on the substrate. The new design and precision fabrication process make it viable to explore more complex microstrip components printed on the substrate and higher frequency applications. The proximity of the BPM and the camera $(<5 \mathrm{~cm})$ will provide a precise calibration platform to study shot-toshot jitter, long-term stability of both systems, and the dependence of BPM signal on beam properties (size, charge distribution) due to nonlinearity.
\end{abstract}

\section{INTRODUCTION}

A chicane bunch compressor was designed and implemented at the Advanced Photon Source (APS) in 2000 [1] to increase the peak current of the bunch and improve the performance of the low-energy undulator test line (LEUTL) free-electron laser (FEL). It is expected to operate at $\sim 200 \mathrm{MeV}$ with normalized emittance in the range of $1 \pi$ to $4 \pi \mathrm{mm}$ mrad. Coherent synchrotron radiation (CSR) effects are expected to be significant at these emittance levels. Their study calls for accurate emittance measurement at the level of several percent or better.

TABLE 1. Electron Beam Parameters at the Three Screen Section

ELECTRON ENERGY (MEV)

Single bunch charge $(\mathrm{nC})$

Normalized emittance $(\pi \mathrm{mm} \cdot \mathrm{mrad})$

Emittance $\varepsilon(\pi \mathrm{mm} \cdot \mathrm{mrad})$

Beta function at beam waist $\beta$ (m)

Rms beam size, $\sqrt{\varepsilon \beta}(\mu \mathrm{m})$

Rms beam size, $\sqrt{\varepsilon / \beta}$ ( $\mu \mathrm{rad})$

\section{$200(\gamma=400)$}

$0.2-1.0 \mathrm{x}$

$\begin{array}{ll}4.0 & 1.0\end{array}$

$0.010 \quad 0.0025$

1.00

100

50

100 
A compact, modular imaging system [2] was designed and implemented to support studies of the low-emittance beams, with the high resolution, reliability, reproducibility, and accuracy needed. In this paper, we report several implemented and planned upgrades to the system.

A new screen mount was designed and installed. It includes a wakefield shield, a YAG screen mount, an optical transition radiation (OTR) screen mount, and a calibration target. A new set of optics was designed to focus in the far field, or image the angular divergence of the electron beam when the OTR screen is employed.

A compact electronic beam position monitor (BPM) was designed to mount directly on the flag. The BPM design uses a new fabrication process, which is expected to make it more viable to explore complex electrode configurations and for higher frequency applications.

\section{FOUR-POSITION SCREEN MOUNT}

Due to high-energy spread used for chirping the beam, the rms beam size in the horizontal direction can reach several millimeters in the midsection of the chicane. This is comparable to the aperture of the vacuum vessel of $25 \mathrm{~mm}$. A smooth transition of the beam pipe is desired to reduce wakefield effects to the electron bunch. Figure 1 shows the design of the wakefield shield. Figure 2 shows an ACAD rendering of the screen mount assembly.

The wakefield shield / transition is mounted at position no. 1 on a four-position vacuum feedthrough, powered by a pair of pneumatic actuators connected back-toback. The stroke of the short actuator is $38.4 \mathrm{~mm}$ and that of the long actuator is 76.4 $\mathrm{mm}$. Four screen positions (38.4 mm spacing) can be reached by extending or contracting these two pneumatic actuators.

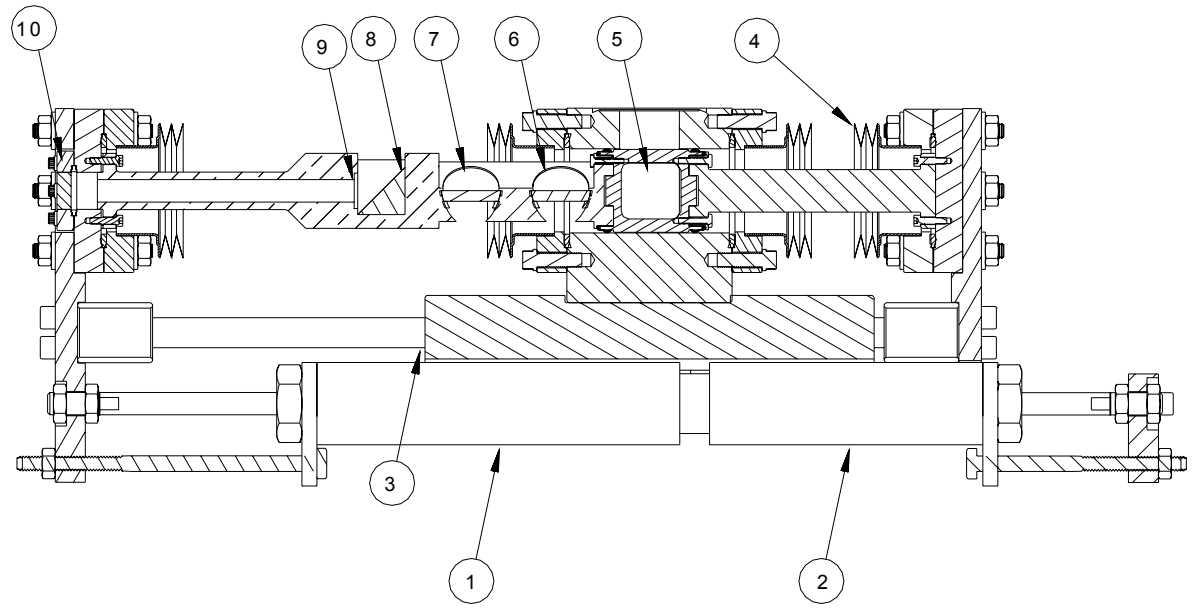

FIGURE 1. Four-position screen mount assembly: (1) Long-stroke actuator, (2) short-stroke actuator, (3) mounting block and linear bearing housing, (4) vacuum bellow, (5) wakefield shield / transition, (6) OTR screen mount, (7) YAG screen mount, (8) mirror, (9) calibration target, and (10) viewport for target illumination. 


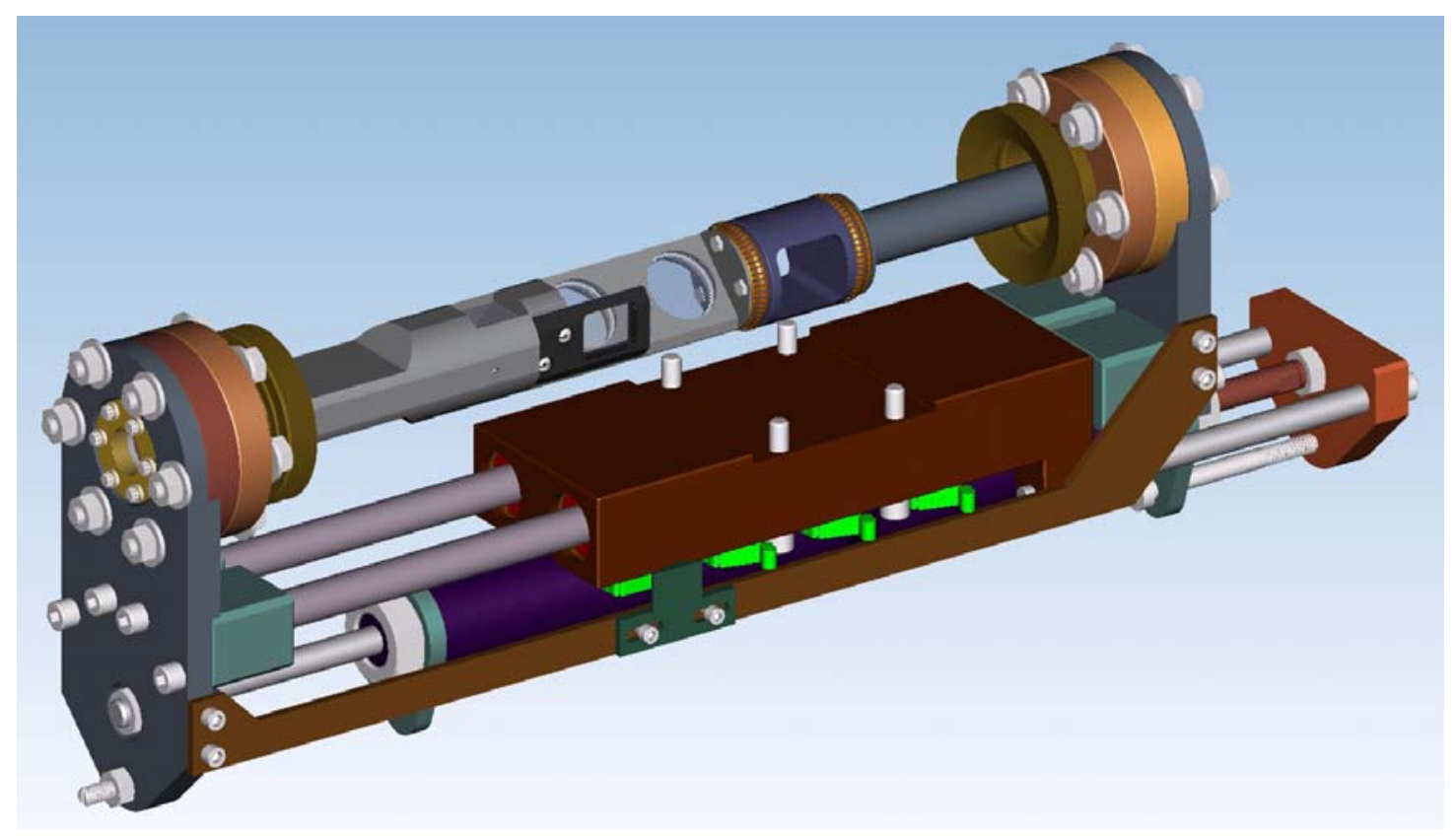

FIGURE 2. ACAD 3-D rendering of the four-position screen mount assembly.

At position no. 2, a 45-degree mirror is mounted as an OTR screen. It is used for high-intensity (charge density) electron beams. At position no. 3, a YAG scintillator screen $(20 \mathrm{~mm} \times 15 \mathrm{~mm} \times 0.1 \mathrm{~mm})$ is mounted with a mirror. It is used for lowintensity electron beams.

At position no. 4, an aluminum-coated prism is used as a first surface mirror, in conjunction with a calibration target. The target is designed to have a dark metallic coating with an etched dot matrix at $0.25 \mathrm{~mm}$ spacing. The illumination light is fed from outside of the vacuum through a viewport. Images of these bright dots in the dark background are convenient for the video digitizer to pick out and perform profile analysis. The center coordinates of the dots are used to calibrate pixel sizes, while the rms image size of these $10-\mu \mathrm{m}$-diameter dots are used to obtain optical resolution of the camera. Since the optical distance from the calibration target is the same as from the YAG screen, the target is also used as a focusing aid.

\section{OPTICS FOR BEAM DIVERGENCE MEASUREMENT}

By design, the camera module has two cameras sharing the light with a beam splitter. Normally, each camera has its own imaging optics, one is used for highresolution imaging but covers only a part of the screen, while another is used for lowresolution imaging and covers the full view of the screens. By reconfiguring the optics, however, one camera could be equipped with optics for the far field, i.e., with object space at infinity. 


\section{Measurement Using OTR Angular Distribution}

To measure relatively large beam divergence $\left(\sigma_{\mathrm{x}^{\prime}} \sim 0.1 / \gamma\right)$, we can use the features in the angular distribution of the optical transition radiation. A linear polarizer installed in the turntable can be used to select the direction of polarization to be along the $\mathrm{x}$ or $\mathrm{y}$-axis. When the $\mathrm{x}$-polarization is selected, the OTR angular distribution is,

$$
I_{x}\left(\theta_{x}, \theta_{y}\right)=\frac{2 A}{\pi} \frac{\theta_{x}^{2}}{\left(\gamma^{-2}+\theta_{x}^{2}+\theta_{y}^{2}\right)^{2}},
$$

where $\left(\theta_{\mathrm{x}}, \theta_{\mathrm{y}}\right)$ is the angle from the specular direction, and $A$ is a constant. For an electron beam with Gaussian divergence distribution, with rms divergence of $\left(\sigma_{\mathrm{x}^{\prime}}, \sigma_{\mathrm{y}^{\prime}}\right)$,

$$
g\left(\theta_{x}, \theta_{y}\right)=\frac{1}{2 \pi \sigma_{x^{\prime}} \sigma_{y^{\prime}}} e^{-\frac{\theta_{x}^{2}}{2 \sigma_{x^{\prime}}{ }^{2}}-\frac{\theta_{y}{ }^{2}}{2 \sigma_{y^{\prime}}{ }^{2}}},
$$

the observed spatial distribution is

$$
\int_{-\pi}^{\pi} \int_{-\pi}^{\pi} g\left(\theta_{x}-\theta_{x}{ }^{\prime}, \theta_{y}-\theta_{y}{ }^{\prime}\right) I_{x}\left(\theta_{x}{ }^{\prime}, \theta_{y}{ }^{\prime}\right) d \theta_{x}{ }^{\prime} d \theta_{y}{ }^{\prime} .
$$

If we integrate over the y-coordinates to improve statistics, the resultant profile is

$$
F_{x}\left(\theta_{x}\right)=\int_{-\pi}^{\pi} \int_{-\pi}^{\pi} \int_{-\pi}^{\pi} g\left(\theta_{x}-\theta_{x}{ }^{\prime}, \theta_{y}-\theta_{y}{ }^{\prime}\right) I_{x}\left(\theta_{x}{ }^{\prime}, \theta_{y}{ }^{\prime}\right) d \theta_{x}{ }^{\prime} d \theta_{y}{ }^{\prime} d \theta_{y} .
$$

For $\gamma>>1$, we could change the limits of the integration to infinity. Inserting Eqs. (1)

- (3) into (4), one obtains the following expression,

where

$$
F_{x}(p)=\frac{A}{\sqrt{2 \pi} \sigma_{p}} \int_{-\infty}^{\infty} \frac{q^{2}}{\left(1+q^{2}\right)^{\frac{3}{2}}} e^{-\frac{(q-p)^{2}}{2 \sigma_{p}^{2}}} d q
$$

$$
p=\gamma \theta_{x}, \text { and } \sigma_{p}=\gamma \sigma_{x^{\prime}} .
$$

For very small beam divergence, Eq. (5) gives

$$
F_{x}(0) \square A \sigma_{p}^{2},\left(\sigma_{p}<<1\right) .
$$

The entire profile can be calculated with a given beam divergence $\sigma_{x^{\prime}}$ (Fig. 3). However, for a quick estimate of the beam divergence, the ratio of the center minimum and the maximum of the angular distribution can be used,

$$
\eta \equiv \frac{F_{\min }}{F_{\max }}=\frac{F(0)}{F_{\max }} .
$$

The ratio for Gaussian beams with $\sigma_{p}<<1$ can be easily modeled. The results can be summarized in the following relations,

$$
\gamma \sigma_{x} \equiv \sigma_{p} \cong \sqrt{0.387 \eta+0.467 \eta^{2}+1.135 \eta^{3}}, \quad(\eta \leq 0.7) .
$$

This fitted expression has an accuracy of $1 \%$ or better for $\sigma_{x}<0.8 / \gamma($ Fig. 4). 


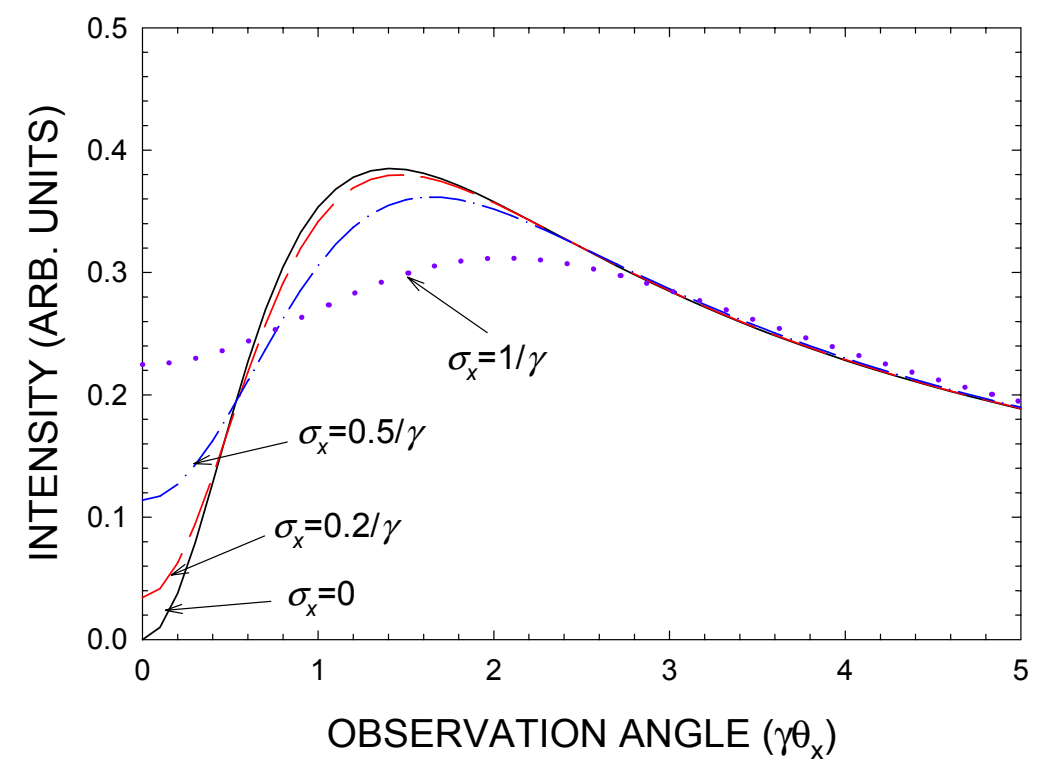

FIGURE 3. Minimum/maximum ratio of the 1-D OTR profile for a Gaussian electron beam.

For our application at the APS bunch compressor, the dynamic range of the system is limited by the video digitizer, which has an 8-bit resolution at zero noise. If we set the noise level at 2 bit, the usable dynamic range is about 6 bit. Best measurements can obtain a ratio of $\eta>2^{-6}$, hence the lowest measurable beam divergence is given by

$$
\sigma_{x} \geq \frac{\sqrt{2^{-6}}}{\gamma}=\frac{1}{8 \gamma},
$$

or $0.3 \mathrm{mrad}$ for $\gamma=400$. Comparison with Table 1 shows that higher resolution will be needed for the measurements at the APS bunch compressor.

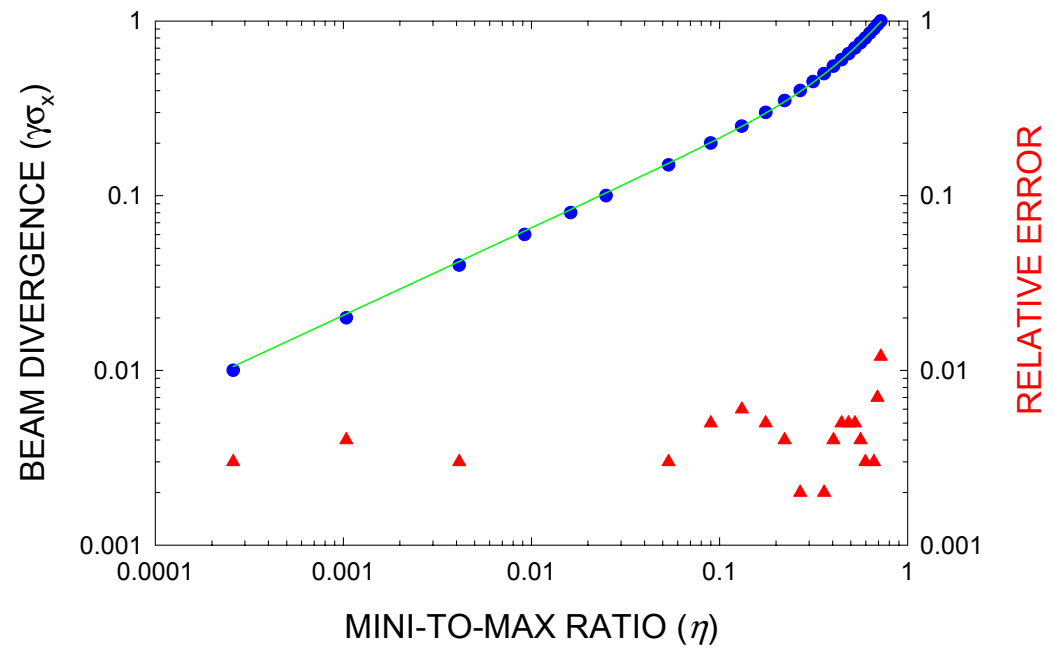

FIGURE 4. The minimum/maximum ratio of the 1-D OTR profile for a Gaussian electron beam, circles: calculated ratio for a given beam divergence, curve: fit by Eq. (9), and triangles: the relative error in beam divergence between the exact calculation and the fit. 


\section{Measurement Using OTR Interferometer}

To improve the resolution of the beam divergence measurements $\left(\sigma_{\mathrm{x}^{\prime}}<0.1 / \gamma\right)$, we could use the OTR interferometer [3]. One thin foil is to be inserted at the first station of the three-screen section, and an OTR screen/mirror is inserted at the mid-station, $1.0 \mathrm{~m}$ downstream from the foil. The far-field camera located at the second station records the interferogram.

To illustrate the resolution of the interferometer at this geometry, we calculated the OTR intensity distribution for circular electron beam $\sigma_{x^{\prime}}=\sigma_{y^{\prime}}=\sigma$. The result is given by a simple integral,

$$
K(\theta)=4 A \int_{0}^{\pi} \int_{0}^{2 \pi} \frac{q^{3}}{\left(1+q^{2}\right)^{2}} \cos ^{2} \frac{\pi\left(1+q^{2}\right)}{p_{\lambda}^{2}} G(\gamma \theta, q) d q,
$$

where $\sigma_{p}=\gamma \sigma, p_{\lambda}=\gamma \sqrt{2 \lambda / L}$ and

$$
G(p, q)=\frac{1}{\pi \sigma_{p}^{2}} \int_{0}^{\pi} \exp \left\{-\frac{1}{2 \sigma_{p}^{2}}\left(p^{2}+q^{2}-2 p q \cos \phi\right)\right\} d \phi .
$$

Assuming we have 1 meter foil-mirror spacing, and an observation wavelength of 0.63 $\mu \mathrm{m}$, we show several sample calculations in Fig. 5, including the beam conditions listed in Table 1. From the simulated interferogram, we conclude that the geometry is suitable for the measurement of beams with divergence less than $0.1 / \gamma$.

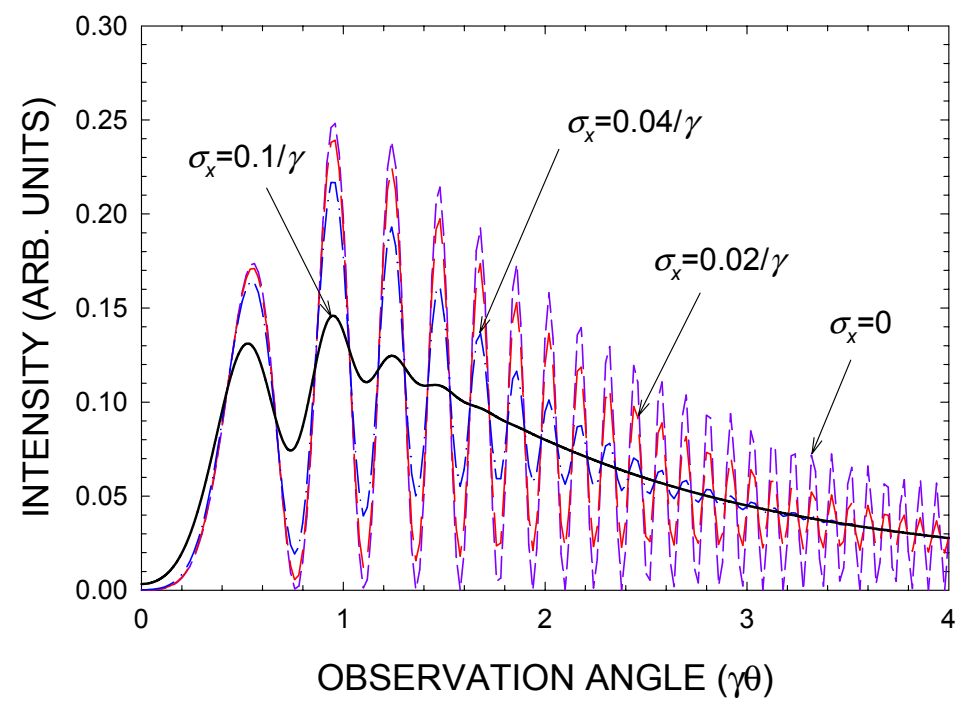

FIGURE 5. The OTR interferometer light intensity as a function of observation angle (in far field). Beam with lower emittance $\left(\sigma_{x}=0\right)$ generate intensity oscillations that extends to higher angles, while beam with higher emittance $\left(\sigma_{x}=0.1 / \gamma\right)$ generates oscillations that damps in a short range.

\section{COMPACT ELECTRONIC BEAM POSITION MONITOR}

The shorted $\lambda / 4$ wave S-band BPM electrode shown in Fig. 6 was designed as a test vehicle for evaluating the performance of a new compact BPM electrode. The goal 
was to reduce the size and assembly complexity for the BPM, so a flag and BPM combination can be used in a limited length of beam line. This concept of combining diagnostics also has the advantage of using a cross calibration scheme that can be implemented to verify the performance of each of the instruments. The proximity of the BPM and the camera $(<5 \mathrm{~cm})$ will give us a good opportunity to study, to micron precision, the dependence of BPM signal on beam properties (size, charge distribution) due to nonlinearity. It also provides a precise calibration platform to study shot-to-shot jitter, and long-term stability of both systems.

Several design features can be seen from Fig. 6: (1) The rf microstrip circuit and vacuum feedthrough connectors are replaceable. This is achieved by welding the feedthrough connectors to a standard removable vacuum flange. The center conductors make contact to the stripline via a beryllium copper spring contact. This design simplifies assembly of the BPM and enables the replacement of a connector in the event of damage. (2) The BPM electrodes are fabricated on a machinable (MACOR) ceramic cylinder substrate with a dielectric constant of 4.88 . The rf circuit is etched on the copper/ceramic substrate with a process similar to printed circuit-board technology. The ceramic cylinder is captivated in the BPM housing by a threaded transition sleeve. The new design and precision fabrication process make it viable to explore more complex microstrip components printed on the substrate and higher frequency applications. The fabrication costs are expected to be much lower than those for the $34 \mathrm{~mm}$ BPM currently in use in the APS linac.

The connectors followed by a bore in the metal housing form 50-ohm transmission lines to each of the four blades. The wall current is intercepted by the 26.24-mm long electrode that forms 50 ohms impedance with the vacuum chamber. The gap that is formed between the vacuum chamber and the stripline is $3.48 \mathrm{~mm}$. The total length of the test BPM stripline assembly is $54.61 \mathrm{~mm}$ and the inner electrode radius $35 \mathrm{~mm}$, which is the same as that for the linac vacuum chamber. The downstream end of the transmission line is electrically shorted, which also provides the mechanical strength and rigidity to support the striplines.

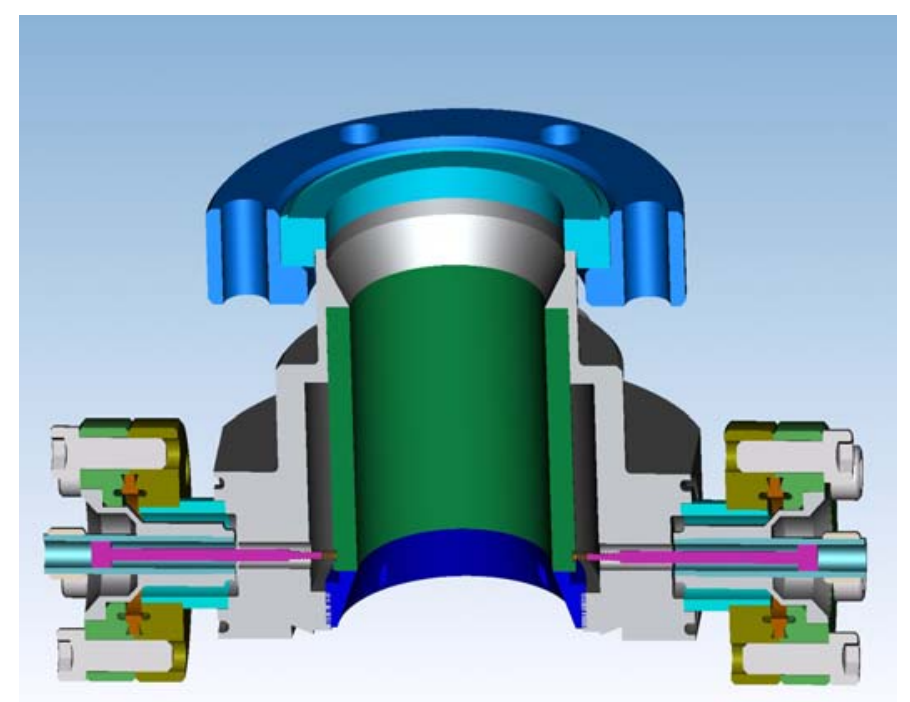

FIGURE 6. Test BPM assembly. 
The preliminary electromagnetic modeling indicates that the ceramic actually improve pincushion distortion performance as compared in Fig. 7. The thickness of the ceramic has not been optimized and is presently set to $3.4 \mathrm{~mm}$ for mechanical reasons. The overall performance is expected to be similar to the standard S-band [3], which is presently used in all APS linac applications.
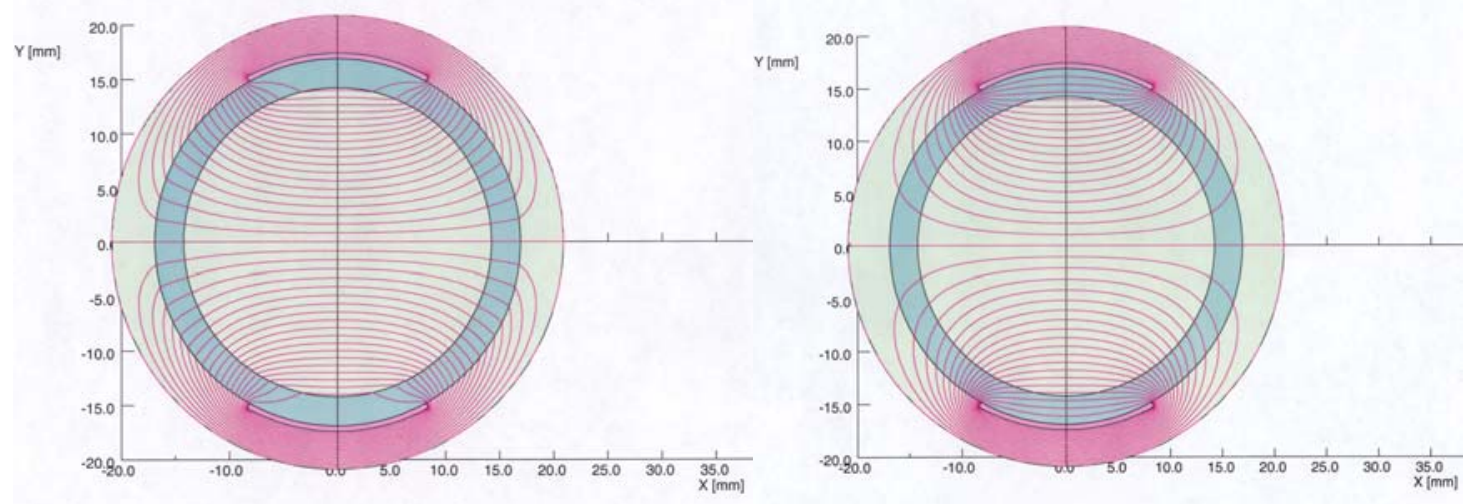

FIGURE 7. Vertical plane equipotential lines with ceramic (left) and without ceramic (right), showing larger linear region in the case of ceramic housing (field lines being parallel and equal spacing).

\section{ACKNOWLEDGMENTS}

We wish to thank G. Decker, S. Milton, M. Borland, and O. Singh for helpful discussions, encouragement, and support.

\section{REFERENCES}

1. Borland, $\mathrm{M}$ et al., A Highly Flexible Bunch Compressor for the APS LEUTL FEL, Proc. LINAC 2000.

2. Yang, B. X., et al., Design and Performance of a Compact Imaging System for the APS Linac Bunch Compressor, Proc. Particle Accelerator Conference 2001, 2335 (2002).

3. R. B. Fiorito and D. W. Rule, "Optical Transition Radiation Beam Emittance Diagnostics", in AIP Conference Proceedings No. 319, R. Shafer, editor (1994).

4. A. Gorski, R. Lill, "Construction and Measurement Techniques for the APS LEUTL Project RF Beam Position Monitors," Proceedings of the 1999 Particle Accelerator Conference, New York, pp. 1411-1413 (1999). 